\title{
Corrections to the Paper by M.Yu. Petukhov and Yu.V. Petukhov* "Parametric Generation of Acoustic-Gravity Waves by Alfvén Waves in the Solar Atmosphere," Astron. Lett. 28, 335 (2002)
}

When interpreting the physical meaning of the two groups of terms with the coefficients $B_{1}, B_{1}^{*}$ and $B_{2}, B_{2}^{*}$ in expression (41) for the vertical oscillation velocity in an acoustic-gravity wave at a difference frequency, we made a mistake. Actually, our explanations for the terms with $B_{1}, B_{1}^{*}$ should refer to the terms with $B_{2}, B_{2}^{*}$; in turn, the explanations for the latter should refer to the terms with $B_{1}, B_{1}^{*}$. Therefore, we made the following erroneous assertions in the text of our paper.

On page 387, in the left column from line 1 to line 3 from the top, we incorrectly assert that "... in the same direction (the terms with $B_{1}$ and $B_{1}^{*}$ in (41)) and in opposite directions (the terms with $B_{2}$ and $B_{2}^{*}$ in (41))." The correct version should be "... in opposite directions (the terms with $B_{1}$ and $B_{1}^{*}$ in (41)) and in the same direction (the terms with $B_{2}$ and $B_{2}^{*}$ in (41))."

Therefore, on page 388, in the right column in lines 21 and 22 from the bottom, and on page 390, in the right column in lines 1 and 2 from the bottom, we incorrectly assert "... in opposite directions...." The correct version should be "... in the same direction...."

Thus, on page 388 , in the right column from line 20 to line 26 and on page 390 in the right column from line 1 to line 6 from the bottom, the same phrase that constitutes one of our conclusions should be correctly read as follows: "... the nonlinear interaction of Alfvén waves propagating in the same direction is the predominant parametric generation mechanism of AGWs at the difference frequency."

This correctly formulated conclusion that follows from our analytical and numerical results is no longer consistent with the conclusions of Wentzel (1974) and the assertions made on their basis by Kaplan et al. (1977) and Priest (1982) that the most favorable conditions for the nonlinear transformation of
Alfvén-wave energy into acoustic-wave energy arise only in the presence of oppositely directed flows of nonlinearly interacting Alfvén waves. This contradiction stems from the fact that Wentzel (1974) considered the nonlinear interaction of plane Alfvén waves in a homogeneous medium, because he disregarded the increase in their velocity with height, which is inversely proportional to the square root of the decreasing density of the solar atmosphere. It is easy to verify that the following assertion results from this simplified statement of the problem in the paper by Wentzel (1974): acoustic waves at a relatively low difference frequency can be generated by counterpropagating Alfvén waves only at a ratio of the speed of sound to the Alfvén-wave velocity smaller than unity, and unidirectional Alfvén waves can generate acoustic waves only at zero difference and sum frequencies.

Taking into account the continuous stratification of the Alfvén-wave velocity automatically gives rise to oppositely propagating and linearly interacting (coupled) Alfvén waves at each point of the medium. For these waves, the dispersion relations for plane waves used by Wentzel (1974), Kaplan et al. (1977), and Priest (1982) no longer hold. Therefore, the relations derived by Wentzel (1974) cannot be used to properly study the parametric generation of acoustic-gravity waves (see, e.g., Kaplan et al. 1977; Priest 1982).

\section{REFERENCES}

1. S. A. Kaplan, S. B. Pikelner, and V. N. Tsytovich, Physics of Solar Atmospheric Plasma (Nauka, Moscow, 1977) [in Russian].

2. E. Priest, Solar Magnetohydrodynamics (Reidel, Dordrecht, 1982; Mir, Moscow, 1985).

3. D. G. Wentzel, Solar Phys. 39, 129 (1974).

*E-mail: petukhov@hydro.appl.sci-nnov.ru 